\title{
Baryon spectroscopy in the unquenched quark model
}

\author{
Roelof Bijker*, Gustavo Guerrero-Navarro and Emmanuel Ortiz-Pacheco \\ Instituto de Ciencias Nucleares, UNAM, AP 70-543, Mexico DF, Mexico \\ E-mail: bijker@nucleares unam.mx, \\ gustavohazel@ciencias.unam.mx, \\ emmanuelo@ciencias.unam.mx
}

We discuss some applications of the unquenched quark model which is an extension of the CQM that includes the effects of sea quarks via a ${ }^{3} P_{0}$ quark-antiquark pair-creation mechanism. Particular attention is paid to the electromagnetic couplings and $\beta$ decays of baryons. It is shown that the observed discrepancies between the experimental data and the predictions of the CQM can be accounted for in large part by the effects of sea quarks in the unquenched quark model.

XVII International Conference on Hadron Spectroscopy and Structure

25-29 September, 2017

University of Salamanca, Salamanca, Spain

\footnotetext{
* Speaker.
} 


\section{Introduction}

The constituent quark model (CQM) describes the nucleon as a system of three constituent, or valence, quarks. Despite the successes of the CQM (e.g. masses, electromagnetic couplings, and magnetic moments), there is compelling evidence for the presence of sea quarks from other observables such as the observed flavor asymmetry of the proton, the proton spin crisis, and the systematics of strong decays of baryons.

In the CQM, baryons are described in terms of a configuration of three constituent (or valence) quarks neglecting the effects of pair-creation (or continuum couplings). Above threshold these couplings lead to strong decays and below threshold to virtual higher-Fock components $(q q q-q \bar{q})$ in the baryon wave function. The effects of these multiquark configurations (or sea quarks) can be studied by unquenching the CQM.

In this contribution, we study the importance of sea quarks for the electromagnetic and weak couplings of baryons.

\section{Unquenched quark model}

In the unquenched quark model (UQM), the effects of sea quarks are included via a ${ }^{3} P_{0}$ quarkantiquark pair-creation mechanism $[1,2,3,4,5]$. The pair-creation mechanism is inserted at the quark level and the one-loop diagrams are calculated by summing over a complete set of intermediate baryon-meson states. As a result, the baryon wave function is given by the sum of a contribution of the valence quarks $|A\rangle$ and a higher-Fock component consisting of an intermediate baryon-meson configuration $|B C\rangle$

$$
\left|\psi_{A}\right\rangle=\mathscr{N}_{A}\left\{|A\rangle+\gamma \sum_{B C l J} \int d \vec{K} k^{2} d k|B C, l, J ; \vec{K}, k\rangle \frac{\left\langle B C, l, J ; \vec{K}, k\left|T^{\dagger}\left({ }^{3} P_{0}\right)\right| A\right\rangle}{\Delta E_{A \rightarrow B C}(k)}\right\},
$$

where the energy denominator is calculated in the rest frame of baryon A

$$
\Delta E_{A \rightarrow B C}(k)=m_{A}-\sqrt{m_{B}^{2}+k^{2}}-\sqrt{m_{C}^{2}+k^{2}} .
$$

Here $\vec{k}$ and $l$ denote the relative radial momentum and relative orbital angular momentum of the baryon-meson system BC. The operator $T^{\dagger}$ creates a quark-antiquark pair in the ${ }^{3} P_{0}$ state with the quantum numbers of the vacuum $[4,5,6]$

In the UQM, the matrix elements of an observable $\hat{\mathscr{O}}$ are calculated as $\left\langle\psi_{A}|\hat{\mathscr{O}}| \psi_{A}\right\rangle$ which is the sum of a contribution from the valence part and from the continuum component (or sea quarks). Previous studies have shown that whereas the effects of quark-antiquark pairs do not change the good results of the CQM for the magnetic moments [4], they provide important contributions to observables like the orbital angular momentum in the spin of the proton [4], the flavor asymmetry of the proton [5], strangeness content of nucleon electromagnetic form factors [3, 7], strangeness suppression [8], and self-energy corrections to baryon masses [9]. In the next section, we discuss the importance of higher-Fock components in electromagnetic and weak decays of baryons. 


\begin{tabular}{cccc}
\hline \hline & CQM & UQM & Exp [12] \\
\hline$\Gamma(\Delta \rightarrow N \gamma)$ & 399 & 608 & $703 \pm 61$ \\
$\pi$ & & 554 & \\
$\pi, \mathrm{K}$ & & 582 & \\
$\pi, \mathrm{K}, \eta, \eta^{\prime}$ & & 608 & \\
\hline \hline
\end{tabular}

Table 1: Radiative decay widths of the $\Delta$ resonance in $\mathrm{keV}$.

\section{Results}

In this section, we discuss some recent results for electromagnetic and weak couplings. A more detailed account will be given in future publications $[10,11]$. In the present calculation, the sum over intermediate states is limited to octet and decuplet baryons in combination with pseudoscalar mesons. The contributions of radially excited baryons and mesons are not taken into account.

\subsection{Electromagnetic decays}

Here we consider the effect of sea quarks for the electromagnetic decay of the $\Delta$ resonance. The radiative width for this process can be obtained in terms of a sum over helicity amplitudes

$$
\Gamma(\Delta \rightarrow N \gamma)=\frac{E_{N} p_{\gamma}^{2}}{2 \pi m_{\Delta}} \sum_{\nu>0}\left|A_{v}\right|^{2},
$$

which in turn for ground state baryons is related to the transition magnetic moment $\mu_{\Delta N}$

$$
\sum_{v>0}\left|A_{v}\right|^{2}=4 \pi p_{\gamma} \mu_{\Delta N}^{2}
$$

The results in Table 1 and Fig. 1 show that the coupling to the pion cloud accounts in great part for the observed discrepancy between the quark model value $399 \mathrm{keV}$ and the experimental value $703 \pm 61 \mathrm{keV}$. The contribution of intermediate states containing kaons and $\eta$ mesons is small compared to that of the pions.

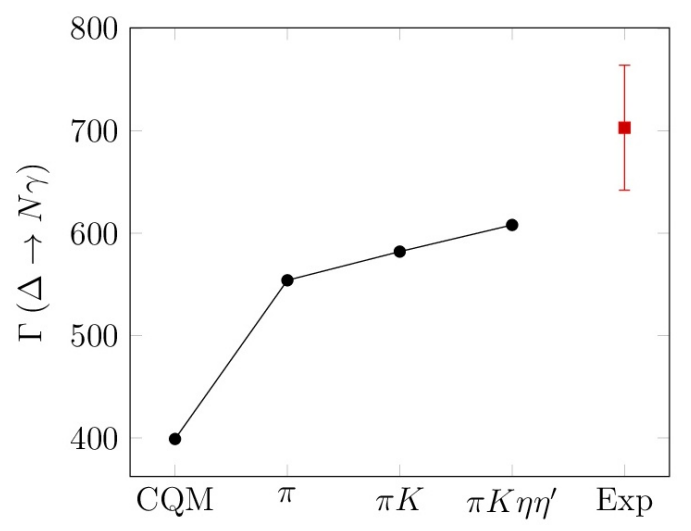

Figure 1: Radiative decay widths of baryons in $\mathrm{keV}: \Gamma(\Delta \rightarrow N \gamma)$. 


\begin{tabular}{cccccc}
\hline \hline$g_{A}$ & \multicolumn{2}{c}{ CQM } & UQM & Cabibbo & Exp [12] \\
\hline$n \rightarrow p$ & $F+D$ & 1.67 & 1.34 & 1.27 & $1.2701 \pm 0.0025$ \\
$\pi$ & & & 1.32 & & \\
$\pi, \mathrm{K}$ & & & 1.35 & & \\
$\pi, \mathrm{K}, \eta, \eta^{\prime}$ & & & 1.34 & & \\
\hline$\Sigma^{-} \rightarrow n$ & $F-D$ & -0.33 & -0.31 & -0.34 & $-0.340 \pm 0.017$ \\
$\pi$ & & & -0.29 & & \\
$\pi, \mathrm{K}$ & & & -0.31 & & \\
$\pi, \mathrm{K}, \eta, \eta^{\prime}$ & & & -0.31 & & \\
\hline & $F$ & $\frac{2}{3}$ & $\frac{2}{3}$ & 0.465 & \\
& $D$ & 1 & 1 & 0.805 & \\
\hline \hline
\end{tabular}

Table 2: Axial couplings of octet baryons.

\subsection{Weak decays}

Semileptonic decay processes of baryons $A \rightarrow A^{\prime}+e^{-}+\bar{v}_{e}$ are described by the axial couplings which for octet baryons can be expressed in terms of $F$ and $D$. In the quark model, they are given by $F=2 / 3$ and $D=1$, and one obtains

$$
\begin{gathered}
g_{A}(n \rightarrow p)=F+D=\frac{5}{3}, \\
g_{A}\left(\Sigma^{-} \rightarrow n\right)=F-D=-\frac{1}{3},
\end{gathered}
$$

compared to the experimental values, $1.2701 \pm 0.0025$ and $-0.340 \pm 0.017$, respectively. In the Cabibbo approach, $F$ and $D$ are determined from the experimental axial couplings, $g_{A}(n \rightarrow p)$ and $g_{A}\left(\Sigma^{-} \rightarrow n\right)$, leading to effective values $F=0.465$ and $D=0.805$. With these values the semileptonic decay processes of baryons are described very well [13].

In Table 2 and Fig. 2 we show a comparison of the results for the axial couplings in the CQM and the Cabibbo approach with those of the unquenched quark model. The contribution of the pions is responsable for a substantial lowering of the neutron axial coupling from the CQM value of 1.67 to 1.32 thus bringing it in much closer agreement with experiment without the need to introduce effective values of $F$ and $D$. On the other hand, the result for the $\Sigma^{-}$hyperon which is described very well in the CQM is hardly changed. This shows that the effective values of $F$ and $D$ used in the Cabibbo approach can be accounted for in large part by the coupling to the pions. A similar conclusion was reached in an earlier study of the meson-cloud model [14].

\section{Summary and conclusions}

In this contribution, we studied the importance of higher-Fock components (or sea quarks) in electromagnetic and weak decays of baryons in the framework of the unquenched quark model. It was shown that the observed discrepancies between the experimental data and the predictions of 

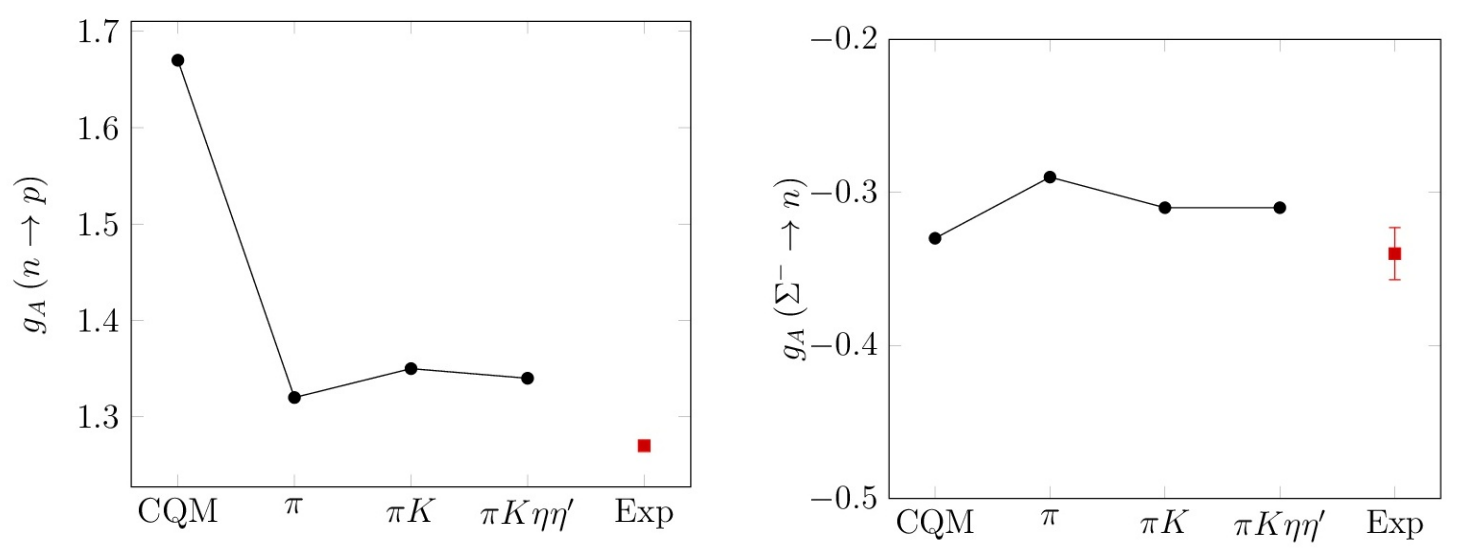

Figure 2: Axial couplings of octet baryons: $g_{A}(n \rightarrow p)$ (left) and $g_{A}\left(\Sigma^{-} \rightarrow n\right)$ (right).

the CQM can be accounted for in large part by the contributions of quark-antiquark pairs in the UQM. In particular, for the decays $\Delta \rightarrow N+\gamma$ and $n \rightarrow p+e^{-}+\bar{v}_{e}$ the contribution of the sea quarks is dominated by the pions.

\section{Acknowledgements}

This work was supported in part by grant IN109017 from DGAPA-UNAM, Mexico and grant 251817 from CONACyT, Mexico.

\section{References}

[1] N.A. Törnqvist, Acta Phys. Polon. B 16, 503 (1985).

[2] P. Zenczykowski, Ann. Phys. (N.Y.) 169, 453 (1986).

[3] P. Geiger and N. Isgur, Phys. Rev. D 55, 299 (1997).

[4] R. Bijker and E. Santopinto, Phys. Rev. C 80, 065210 (2009) [arXiv:0912.4494].

[5] E. Santopinto and R. Bijker, Phys. Rev. C 82, 062202(R) (2010).

[6] W. Roberts and B. Silvestre-Brac, Few-Body Systems 11, 171 (1992).

[7] R. Bijker, J. Ferretti and E. Santopinto, Phys. Rev. C 85, 035204 (2012).

[8] E. Santopinto, R. Bijker and H. García-Tecocoatzi, Phys. Lett. B 759, 214 (2016) [arXiv:1601.06987].

[9] H. García-Tecocoatzi, R. Bijker, J. Ferretti and E. Santopinto, Eur. Phys. J. A 53, 115 (2017) [arXiv:1603.07526].

[10] G. Guerrero-Navarro and R. Bijker, to be published.

[11] E. Ortiz-Pacheco and R. Bijker, to be published.

[12] C. Patrignani et al. (Particle Data Group), Chin. Phys. C 40, 100001 (2016).

[13] N. Cabibbo, E.C. Swallow and R. Winston, Annu. Rev. Nucl. Part. Sci. 53, 39 (2003).

[14] H. Holtmann, A. Szczurek and J. Speth, Nucl. Phys. A 596, 631 (1996). 